University of Nebraska - Lincoln

DigitalCommons@University of Nebraska - Lincoln

Faculty Publications from Nebraska Center for Materials and Nanoscience

Materials and Nanoscience, Nebraska Center for (NCMN)

8-1-2005

\title{
Magnetization reversal in particulate L10 nanostructures
}

\author{
Jian Zhou \\ University of Nebraska - Lincoln \\ Ralph Skomski \\ University of Nebraska-Lincoln, rskomski2@unl.edu \\ Kory D. Sorge \\ University of Nebraska-Lincoln, sorge@physics.fau.edu \\ David J. Sellmyer \\ University of Nebraska-Lincoln, dsellmyer@unl.edu
}

Follow this and additional works at: https://digitalcommons.unl.edu/cmrafacpub

Part of the Nanoscience and Nanotechnology Commons

Zhou, Jian; Skomski, Ralph; Sorge, Kory D.; and Sellmyer, David J., "Magnetization reversal in particulate L10 nanostructures" (2005). Faculty Publications from Nebraska Center for Materials and Nanoscience. 3. https://digitalcommons.unl.edu/cmrafacpub/3

This Article is brought to you for free and open access by the Materials and Nanoscience, Nebraska Center for (NCMN) at DigitalCommons@University of Nebraska - Lincoln. It has been accepted for inclusion in Faculty Publications from Nebraska Center for Materials and Nanoscience by an authorized administrator of DigitalCommons@University of Nebraska - Lincoln. 
Published in Scripta Materialia, Volume 53, Issue 4, August 2005, Pages 453-456.

doi:10.1016/j.scriptamat.2005.04.035 http://www.sciencedirect.com/science/journal/13596462

Copyright (C) 2005 Acta Materialia Inc. Published by Elsevier Ltd. on behalf of Acta Materialia Inc. Used by permission.

\title{
Magnetization reversal in particulate $\mathrm{L}_{0}$ nanostructures
}

\author{
J. Zhou, R. Skomski, K.D. Sorge and D.J. Sellmyer \\ Department of Physics and Astronomy and Center for Materials Research and Analysis \\ University of Nebraska-Lincoln \\ Lincoln, NE 68588, USA
}

Received 1 October 2004; accepted 21 April 2005; available online 23 May 2005; published 1 August 2005.

\begin{abstract}
Magnetization processes in particulate $\mathrm{L}_{0}$ FePt nanostructures are investigated by model calculations and numerical simulations. The systems considered include anisotropic nanograins embedded in non-magnetic matrix; randomly oriented nanoclusters embedded in a $\mathrm{C}$ matrix, and nanocomposites of $\mathrm{FePt}$ particles in a semi-hard matrix. The reversal mechanisms depend on both intra- and intergranular features. Quasi-coherent rotation dominates the reversal in weakly-coupled granular magnets, but interface imperfections yield a disproportionately strong coercivity reduction. Strong intergranular exchange leads to a transition to a discrete pinning regime, which is accompanied by a coercivity maximum.
\end{abstract}

Keywords: Magnetization reversal; L1 0 magnets; Exchange interaction; Discrete pinning

\section{Introduction}

The large uniaxial anisotropy $K_{1}$ of materials such as FePt and $\mathrm{CoPt}$ is the basis for creating coercivity $H_{\mathrm{c}}$ in $\mathrm{L} 1_{0}$-based permanent magnets and magnetic recording media [1], [2] and [3]. However, the corresponding Stoner-Wohlfarth prediction $H_{\mathrm{a}}=2 K_{1} / \mu_{\mathrm{o}} M_{\mathrm{s}}$ significantly overestimates the coercivity, because it ignores the real structure of the magnets [4] and [5]. There are two main reasons for the reduced coercivity: intragranular imperfections and intergranular interactions, including domain-wall pinning effects.

The real-structure dependence of the coercivity, as epitomized by the simultaneous presence of magnetically hard and soft phases, has far-reaching consequences. First, it limits the performance of nanostructured $\mathrm{L}_{0}$ permanent magnets, such as $\mathrm{FePt}: \mathrm{Fe}_{3} \mathrm{Pt}$ [3], and is responsible for the 'residual' deviations from Stoner-Wohlfarth magnetism, as encountered in thin-film FePt dots deposited onto $\mathrm{MgO}$ [6]. Second, in magnetic recording media, the large anisotropy of $\mathrm{L} 10$ particles helps to ensure the thermal stability of the stored information. However, the coupling to a soft phase may reduce the coercivity to facilitate writing, without necessarily deteriorating the zero-field thermal stability of the medium [7].

This paper focuses on the static softening of the hysteresis of interaction-free and interacting $\mathrm{L} 1_{0}$ nanoparticles. The corresponding dynamic effects [4], [5] and [8], being of importance in many of the considered scenarios, including that of Ref. [7], will be discussed elsewhere.

\section{Core-shell nanoparticles}

To a large extent, the physics of inhomogeneous magnetic systems is contained in the core-shell model. Specifically, magnetic recording media tend to contain nanograins that are isolated by a non-magnetic matrix and do not exhibit strong intergranular interactions. (The effects of intergranular magnetostatic and exchange interactions will be analyzed in Sections Sections 3 and 4, respectively.)

Measured coercivities tend to be significantly smaller than the Stoner-Wohlfarth predictions. For example, the 


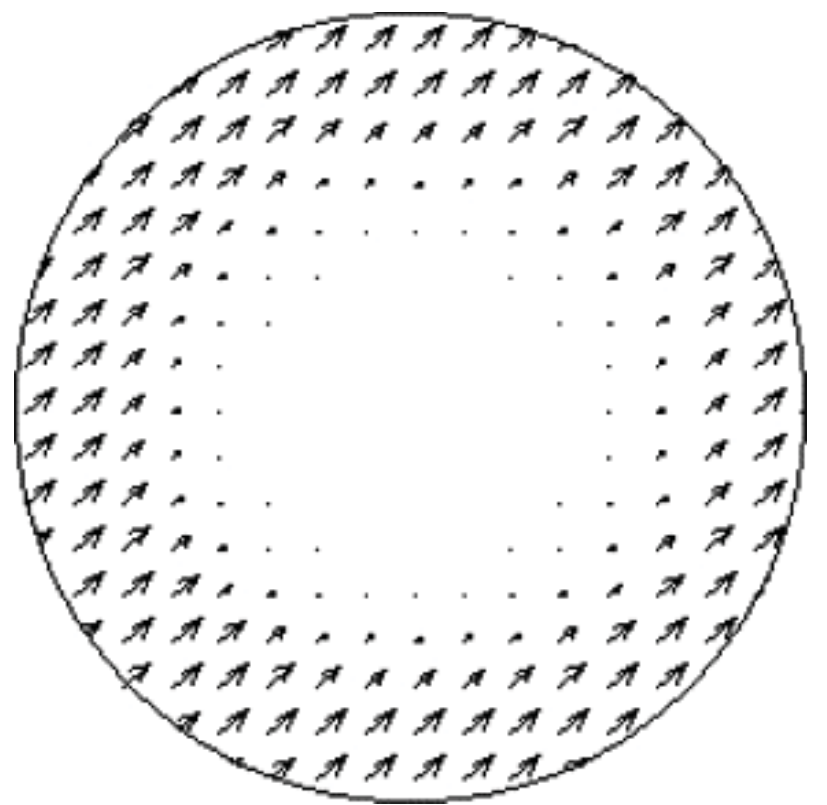

Fig. 1. Real-space spin structure of a core-shell particle $\left(R_{\text {core }}=R-\Delta R\right)$.

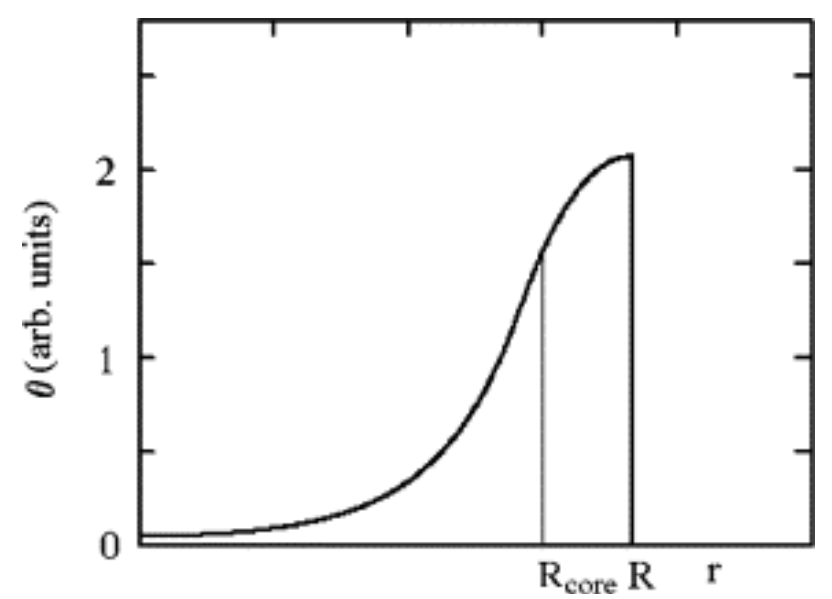

Fig. 2. Analytical results for size-dependence of spheres' spin structure.

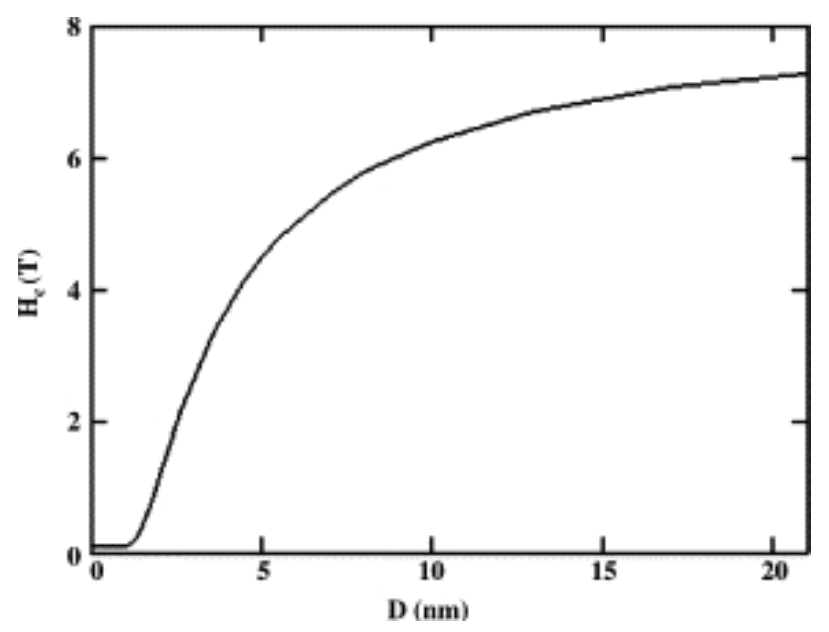

Fig. 3. Coercivity as a function of the particle diameter $D=2 R$ for typical $\mathrm{L}_{0}$ parameters. particles investigated in Ref. [2] have an anisotropy field of $12 \mathrm{~T}$ (or $120 \mathrm{kOe}$ ), but the observed coercivity is only about 2 T. Aside from metallurgical contributions, such as interphase diffusion, this reflects magnetocrystalline surface and interface anisotropy [5], [9] and [10]. Previous research has shown that FePt nanoparticles have different properties at the surface rather than inside the sphere [11]. Very recently [12], analysis has shown that missing $3 \mathrm{~d}-4 \mathrm{~d} / 5 \mathrm{~d}$ bonds at surfaces and interfaces yield a disproportionately strong reduction of the anisotropy, similar to the effect of finite-temperature disorder [13].

In this section, we consider magnetic particles characterized by reduced surface anisotropy. Nucleation-field analysis, that is, considering perpendicular magnetization modes $\mathbf{m}$ of the type $\mathbf{M}=M_{\mathrm{s}}\left(\sqrt{1-m^{2}} \mathbf{e}_{\mathrm{z}}+m\right)$, yields [5]

$$
\nabla^{2} m+\kappa(r)^{2} \mathbf{m}=0
$$

where $\kappa^{2} \quad(r)=\left(K_{1}(r)+\mu_{\mathrm{o}} M_{\mathrm{s}} H / 2\right) / A$. The magnetization modes $m(r)$ are obtained as eigenmodes of Eq. (1) subject to the free surface boundary condition $\mathrm{d} m / \mathrm{d} r=0$. In addition, $A \mathrm{~d} m / \mathrm{d} r$ and $m$ are continuous inside the material [14], but since $A$ is much less real-structure dependent than $K_{1}$ it is usually sufficient to assume that both $\mathrm{d} m / \mathrm{d} r$ and $m$ are continuous.

For spherical geometries, the solutions of Eq. (1) are spherical Bessel functions. The nucleation behavior of stepwise continuous profiles $K_{1}(r)=K_{h}$ for $r<R_{\text {core }}$ and $K_{1}(r)=K_{\mathrm{s}}$ for $R_{\text {core }}<r<R$ amounts to a superposition of functions of the type $\sin (x) / x, \cos (x) / x$, and $\sinh (x) / x$. Fig. 1 and Fig. 2 show the real-space meaning of the perpendicular component $|m|$ and its explicit radial dependence, respectively.

Fig. 3 shows the nucleation-field coercivity for a soft shell of thickness $0.5 \mathrm{~nm}$. The assumed core and shell anisotropies are 5 and $0.05 \mathrm{MJ} / \mathrm{m}^{3}$, respectively. The shallow small-particle minimum indicates the vanishing of the hardmagnetic phase when $\Delta R=R$. For $R=\infty, H_{\mathrm{c}}$ remains smaller than $2 K_{1} / \mu_{\mathrm{o}} M_{\mathrm{s}}$ due to surface nucleation.

Numerically, we have analyzed single particles with core-shell structure using the OOMMF micromagnetic simulation code provided by NIST [15]. The simulations are based on the Landau-Lifshitz-Gilbert equation, and the respective anisotropy, exchange, and magnetization parameters are $K_{1}=6 \mathrm{MJ} / \mathrm{m}^{3}, A=10 \mathrm{pJ} / \mathrm{m}$, and $M_{\mathrm{s}}=1 \mathrm{MA} / \mathrm{m}$. The unit cell size is chosen as $0.75 \mathrm{~nm}$.

One reason for performing the numerical simulations is to investigate the hysteresis-loop shape. In the StonerWohlfarth model, the parallel and perpendicular hysteresis loops reach $M=M_{\mathrm{s}}$ at the same field $H_{\mathrm{a}}$. In reality, both intersection fields are reduced, but the reduction of the coercivity is more pronounced.

Our uniaxial FePt model particles have a core-shell magnetic structure with an outer diameter of $6.7 \mathrm{~nm}$ and a softshell thickness of $0.75 \mathrm{~nm}$. 


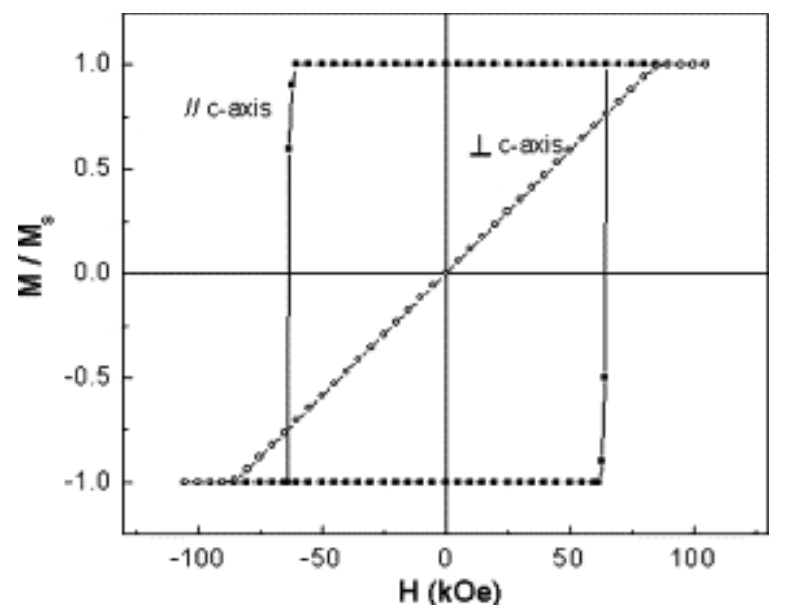

Fig. 4. Hysteresis loops of core-shell structure.

Fig. 4 shows the simulated hysteresis loops for the coreshell sphere. In agreement with experiment, both the effective anisotropy, as given by the perpendicular loop, and the coercivity are smaller than the anisotropy field of $120 \mathrm{kOe}$. The coercivity reduction is particularly pronounced, qualitatively reproducing the hysteresis-loop narrowing frequently encountered [2] and [16] in practice.

Quantitatively, the total coercivity reduction of about $50 \%$ falls in the range encountered in practice, although in many $\mathrm{L} 1_{0}$ systems there is a additional decrease due to difficult-to-quantify factors such as incomplete $\mathrm{L} 1_{0}$ ordering.

\section{Ensembles of nanoparticles}

Even in the absence of intergranular exchange interactions, there is a hysteresis-loop modification due to magnetostatic interactions. In diluted systems, these interactions do not affect the Stoner-Wohlfarth character of the individual grains' reversal but may slightly distort the hysteresis loop.

A good experimental example is diluted FePt nanoclusters in a $\mathrm{C}$ matrix. Recent investigations have yielded a low

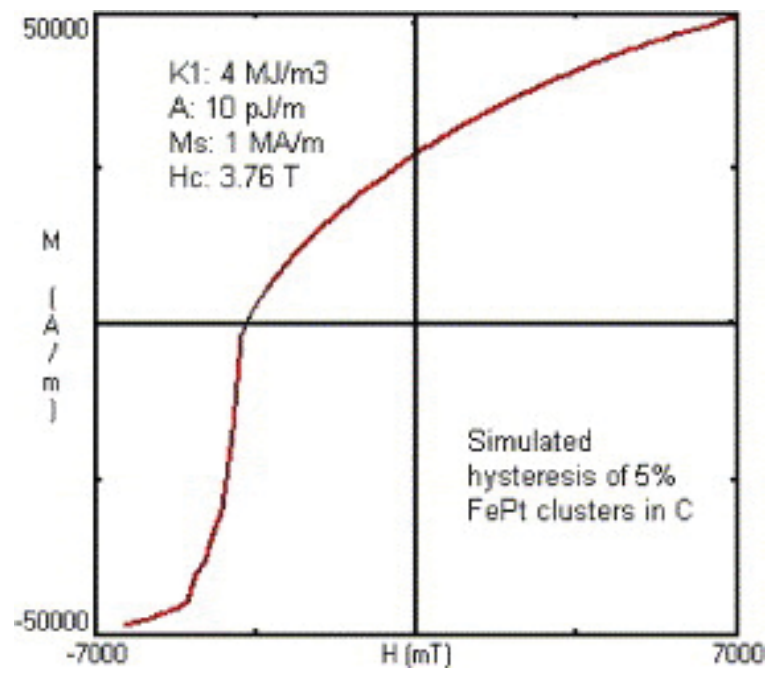

Fig. 5. Simulated hysteresis for assembly of isolated nanoclusters. temperature coercivity of more than $40 \mathrm{kOe}$ in a $5 \% \mathrm{FePt}$ nanoclusters in C matrix [17]. High-resolution transmission electron microscopy (TEM) shows that the clusters are single-crystalline, with a diameter of about $5 \mathrm{~nm}$, and well separated by the $\mathrm{C}$ matrix [17]. In such a condition there is no intergranular exchange interaction.

To simulate this system, $27 \mathrm{Ll}_{0} \mathrm{FePt}$ spheres with diameter of $5 \mathrm{~nm}$ are arranged in a cubic lattice, with a center-to-center distance of $9 \mathrm{~nm}$, corresponding to a 5 vol.\% FePt spheres in $\mathrm{C}$ matrix. The anisotropy axis of each sphere is randomly oriented, representing the randomly distributed easy axis for each FePt cluster. Fig. 5 shows a simulated magnetic hysteresis curve. The measured coercivity at $10 \mathrm{~K}$ is $4 \mathrm{~T}$, as compared to the simulated coercivity of $3.76 \mathrm{~T}$. The curve matches the experimental result well, indicating the diluted FePt nanoclusters reverse as Stoner-Wohlfarth particles.

\section{Dependence of coercivity on exchange}

In nanocomposite systems, the mechanism of magnetization reversal depends on the density of the magnetic particles. With increasing particle density, intergranular exchange interactions become important. Previous work has shown that in the Sm-Co particulate system the coercivity mechanism changes from a coherent rotation regime for non-exchangeinteraction to discrete pinning for large exchange-interaction [18]. Similarly, in the FePt particulate magnets, both analytical and numerical simulations show a coercivity maximum during the transition between the two regimes.

In our numerical simulation, $18 \mathrm{FePt}$ spheres with $7 \mathrm{~nm}$ diameter are arranged in a two-layer rectangular lattice. Fig. 6 shows a typical spin structure during magnetization reversal. Inside the grains, the magnetization is largely coherent, whereas most of the magnetization gradient is localized between the grains. This agrees with earlier analytical calculations [5].

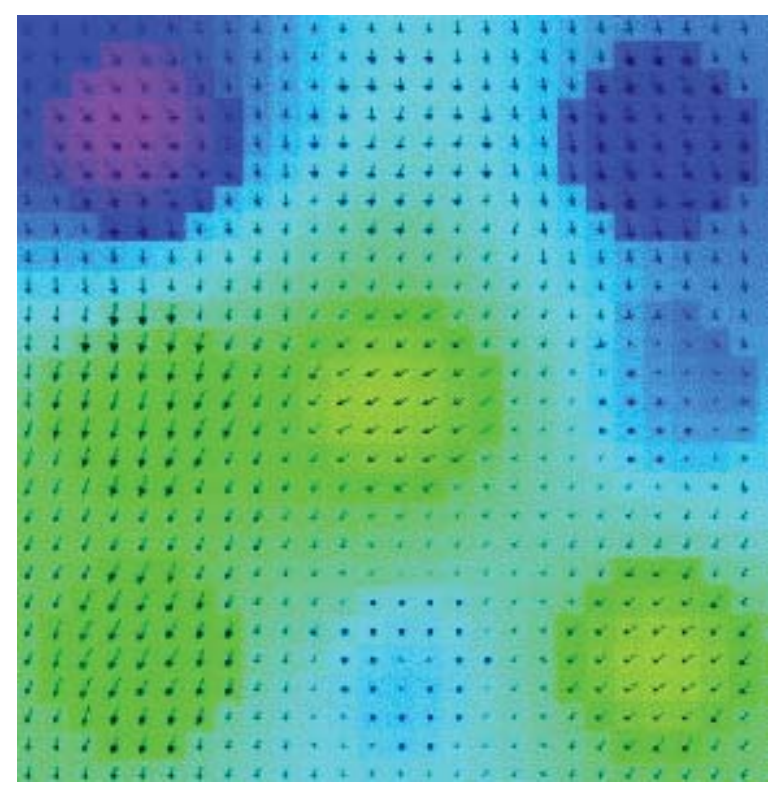

Fig. 6. Spin structure of nano-particulate FePt particles in matrix. 
Fig. 7 shows a typical demagnetization curve for $\mathrm{FePt}$ spheres in a semi-hard matrix, such as FeNiPt. The anisotropies taken are $6 \mathrm{MJ} / \mathrm{m}^{3}$ for FePt particles and $2 \mathrm{MJ} / \mathrm{m}^{3}$ for the matrix. To investigate the dependence of coercivity on the intergranular exchange, the exchange constant $A$ of the matrix is varied. Fig. 8 shows that with an increasing exchange constant $A$, the coercivity of the system reaches a maximum and then decreases. This figure shows that, with increasing exchange, the magnetization reversal transits from a nucleation-type regime to a discrete-pinning regime. Below the coercivity maximum, the reversal is realized by the switching of individual grains, or of small clusters. Above the coercivity maximum, the reversal proceeds by the motion of 'domain walls' separating big interaction clusters [18]

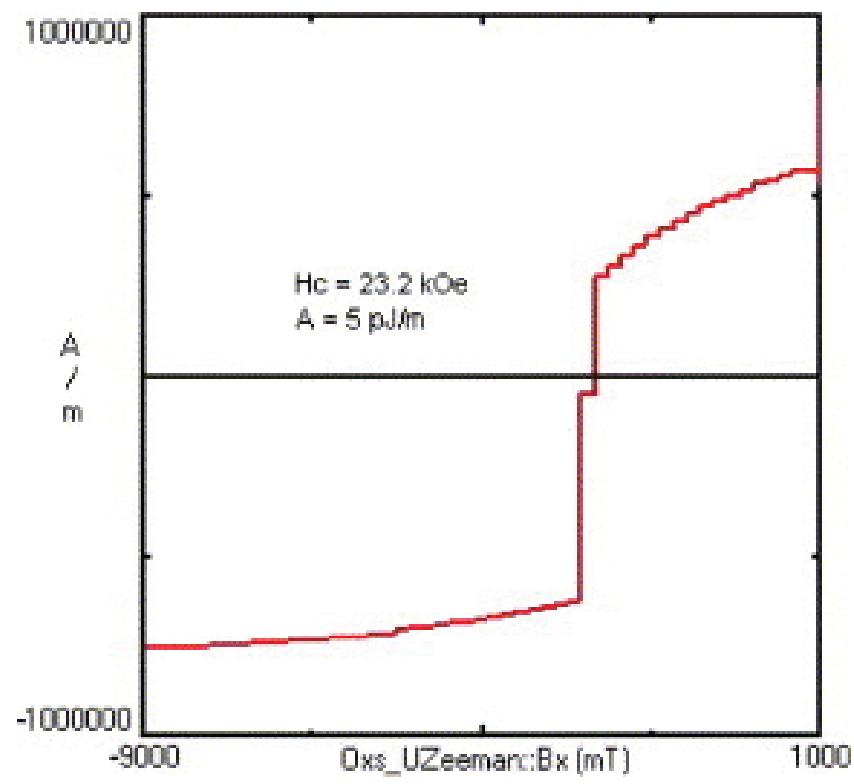

Fig. 7. Simulated hysteresis for the FePt matrix system.

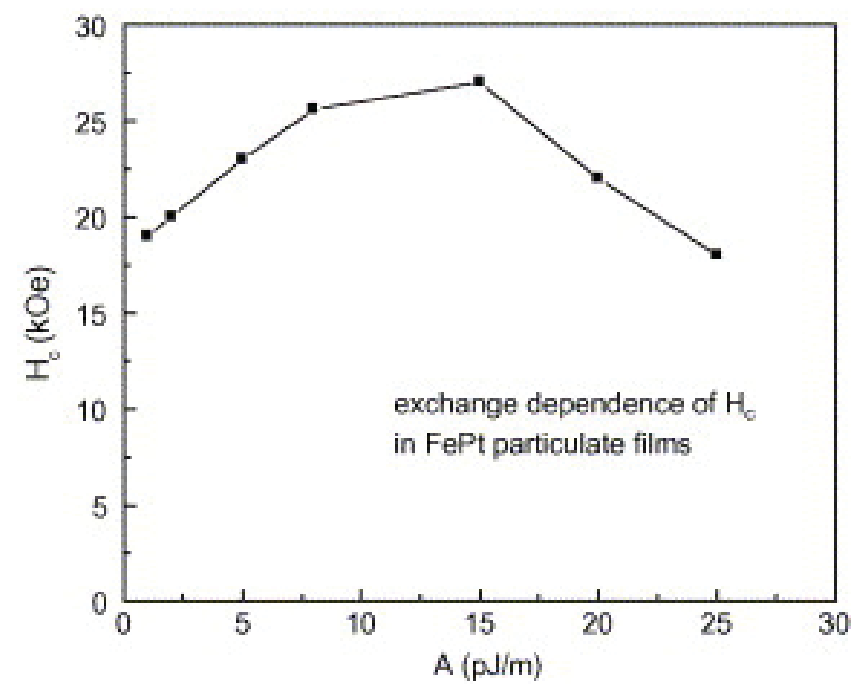

Fig. 8. Exchange-dependence of coercivity of nanocomposites.

\section{Conclusions}

In summary, we performed analytical and numerical micromagnetic calculations to investigate the magnetization reversal in systems of $\mathrm{L} 1_{0} \mathrm{FePt}$ nanograins and clusters; this was investigated by micromagnetic simulations. The experimental systems considered included anisotropic nanograins embedded in non-magnetic matrices such as $\mathrm{C}$, $\mathrm{SiO}_{2}$, and $\mathrm{Ag}$, randomly oriented nanoclusters in a $\mathrm{C}$ matrix, and an exchange-coupled nanocomposite of FePt particles embedded in semi-hard matrix. Both interaction-free and interacting grains have been investigated. The magnetization reversal in weakly-coupled granular magnets is very similar to Stoner-Wohlfarth coherent rotation, but reduced anisotropy due to surface and interface imperfections yields a disproportionately strong coercivity reduction. In FePt nanocomposites, increasing intergranular exchange interactions yield a transition to a discrete pinning regime, where the magnetization remains nearly coherent in any given grain but a domain wall forms between the grains. The transition from single-grain rotation to discrete pinning is accompanied by a coercivity maximum.

\section{Acknowledgements}

This research is supported by NSF-MRSEC, the W. M. Keck Foundation, and CMRA.

\section{References}

[1] H. Zeng, Appl Phys Lett 85 (2004), p. 792. D.J. Sellmyer, Nature 420 (2003), p. 374.

H. Zeng, J. Li, J.P. Liu, Z.L. Wang and S.H. Sun, Nature $\mathbf{4 2 0}$ (2003), p. 395.

[2] M.L. Yan et al., Appl Phys Lett 83 (2003), p. 3332.

[3] P. Liu, C.P. Luo, Y. Liu and D.J. Sellmyer, Appl Phys Lett (1998).

[4] R. Skomski and J.M.D. Coey, Permanent magnetism, IOP, Bristol (1998).

[5] R. Skomski, J Phys: Condens Mater 15 (2003), p. R841.

[6] T. Shima, K. Takanashi and K. Hono, Appl Phys Lett 81 (2002), p. 1050.

[7] R.H. Victora and X. Shen, IEEE Trans Magn 41 (2005), p. 537.

[8] R. Skomski, D. Leslie-Pelecky, R.D. Kirby, A. Kashyap and D.J. Sellmyer, Scripta Mater 48 (2003), p. 857.

[9] W. Scholz, D. Suess, T. Schrefl and J. Fidler, J Appl Phys 95 (2004), p. 6807.

[10] D. Sander, R. Skomski, C. Schmidthals, A. Enders and J. Kirschner, Phys Rev Lett 77 (1996), p. 2566.

[11] H. Kachkachi and H. Mahboub, J Magn Magn Mater 278 (2004), p. 334.

[12] Mryasov O, et al., unpublished.

[13] R. Skomski, A. Kashyap and D.J. Sellmyer, IEEE Trans Magn 39 (2003), p. 2917.

[14] R. Skomski and J.M.D. Coey, Phys Rev B 48 (1993), p. 15812.

[15] http://math.nist.gov/oommf/

[16] V. Karanasos et al., Appl Phys Lett 79 (2001), p. 1255.

[17] Xu YF, Sellmyer DJ. unpublished.

[18] J. Zhou, A. Kashyap, Y. Liu, R. Skomski and D.J. Sellmyer, IEEE Trans Magn 40 (2004), p. 2940. 